\title{
Fuzzy Sets for Human Fall Pattern Recognition
}

\author{
Marina V. Sokolova ${ }^{1,2}$ and Antonio Fernández-Caballero ${ }^{1,3}$ \\ 1 Instituto de Investigación en Informática de Albacete (I3A) \\ Universidad de Castilla-La Mancha, 02071-Albacete, Spain \\ Marina.Sokolova@uclm.es, Antonio.Fdez@uclm.es \\ 2 South-West State University, 94, 50 let Oktyabrya, 305040 - Kursk, Russia \\ 3 Departamento de Sistemas Informáticos, Universidad de Castilla-La Mancha, \\ 02071-Albacete, Spain
}

\begin{abstract}
Vision-based fall detection is a challenging problem in pattern recognition. This paper introduces an approach to detect a fall as well as its type in infrared video sequences. The regions of interest of the segmented humans are examined image by image though calculating geometrical and kinematic features. The human fall pattern recognition system identifies true and false falls. The fall indicators used as well as their fuzzy model are explained in detail. The fuzzy model has been tested for a wide number of static and dynamic falls.
\end{abstract}

\section{Introduction}

Human posture recognition is one of the core problems in computer vision and pattern recognition. It has become a basic component of a greater part of application problems such as ambient intelligence, surveillance, action recognition, human-computer interaction, elderly health care, among others [12]. One particular human posture recognition task is fall detection. A correct and rapid recognition of a fall is essential in assisted living applications. This is specially true in case of elderly people monitoring [10].

There are various fall detection methods. Some make use of wearing devices; other approaches are sensor-based (smart rooms, sensible floors) or process visual data [2]. The first group of methods suppose that a person wears a miniaturized device that collects parameters and launches an alarm in case a fall is detected. A wide type of accelerometers or movement/vibration sensors belong to this group [1]. Although the usage of wearable devices provides many advantages, some authors point out the shortcomings of this approach, namely, reluctance and negligence to wear sensors, and the tendency of wearing devices to produce false alarms [6]. The second group includes various solutions which imply sensors placed in the user's environment. It includes solutions based on floor vibration and acoustic sensing [8], and even on installation of sensitive floor tiles [7], [13]. As these sensors are fixed or integrated into a given environment, they can not easily be moved when a person changes his/her position. In general, acoustic and vibration sensors are expensive and fragile. They may as well request special conditions for a correct functioning. On their side, visual data processing methods are free from the limitations of the previously described groups of methods. This class combines various solutions, such as head motion analysis, shape motion analysis, and inactivity detection [15], [16]. 
In this paper an approach for human fall recognition is introduced. It is based on fuzzy patterns dedicated to detect several fall types in segmented infrared video sequences. The segmentation phase is based on the accumulative computation approach and some infrared spectrum processing algorithms, which segment the original image, detect candidate to human blobs, and define and confirm region of interest (ROI) for segmented human [3]-[5]. The paper focuses on a novel fuzzy model which obtains fall patterns as function of geometrical, temporal and kinematic parameters of humans previously detected in video sequences [18], [11], [14].

\section{Fall Pattern Recognition}

The proposed fuzzy model detects falls and their fall pattern. The objective of the paper is to recognize falls from a "standing" position, from a "sitting" position and from a "lying" position. Indeed, these are the most usual human positions. The proposal includes the indication of the direction (lateral right, lateral left, backward, forward) and the velocity of the fall. The system is capable of detecting false falls which correspond to other human positions such as "kneeling", "crouching" and "squatting".

\subsection{Determination of Fall Indicators}

A fall is determined as dropping or freely coming down under the influence of gravity, and is described by several parameters that describe the spatial and temporal position of a person in a video sequence. We consider that the parameter fall time, $t_{f}$, lies in the interval ranging from 1 to 3 seconds [17]. For $n$ consecutive ROIs corresponding to a same human (in $n$ consecutive images of a sequence), let $y_{t r, 1}$ and $y_{d l, 1}$ be the top-right and down-left Y-coordinates of the ROI in the first image, and let $y_{t r, n}$ and $y_{d l, n}$ be the top-right and down-left corner Y-coordinates of the ROI in the last ( $n$-th) image, respectively. In the same sense, let $x_{t r, 1}$ and $x_{d l, 1}$ be the top-right and down-left $\mathrm{X}$-coordinates of the ROI in the first image, and let $x_{t r, n}$ and $x_{d l, n}$ be the top-right and down-left corner X-coordinates of the ROI in the $n$-th image. Let us also define $x_{t r}$, $x_{d l}, y_{t r}$ and $y_{l d}$ as the top-right and down-left coordinates of the current ROI. Also, let $w=x_{t r}-x_{d l}$ and $h=x_{t r}-x_{d l}$ be the width and the height, respectively, of the current ROI. The following spatial parameters are considered:

- The width-to-height ratio parameter, $R_{w h}$, is calculated for each ROI. In the case of a standing person $R_{w h}$ it is usually less than a " 0 ". When a person starts loosing an erect position, falling down, or bending, this parameter increases. Our experiments have shown that $R_{w h}$ for a lying person belongs to interval $\in[1.5 \ldots 7.0]$.

$$
R_{w h}=\frac{x_{t r}-x_{d l}}{y_{t r}-y_{d l}}
$$

- The height change parameter, $\Delta h$, is calculated for the first and the last images in a sequence of images stored for a given $t_{f}$. It is a relation of the height of the ROI of the last image to the ROI of the first image.

$$
\Delta h=\frac{y_{t r, 1}-y_{d l, 1}}{y_{t r, n}-y_{d l, n}}
$$


- The fall direction parameter, $d i r_{f}$, is calculated for the first and the last images in a sequence of images stored for a given $t_{f}$. It returns a sign of the difference between the upper left corner of the ROI from the first image and the corresponding coordinate of the ROI from the last image. In case of a negative value, dir $r_{f}$ means "to the left", and in case of a positive value, the $d i r_{f}$ is "to the right".

$$
d i r_{f}=\left\{\begin{array}{l}
1, \text { if }\left(x_{t r, 2}-x_{t r, 1}\right) \geq 0 \\
0, \text { otherwise }
\end{array}\right.
$$

- The position change parameter, $\Delta p$, indicates, in first place, if the upper left corner of the ROI of the last image of the sequence is greater that the corresponding ROI of the first image. Position change is equal to " 1 " in case these statements are true and to " 0 " otherwise. This parameter is introduced in order to facilitate the detection of a fall from a bed, or, in other words, falls from a lying position.

$$
\Delta p=\left\{\begin{array}{l}
1, \text { if }\left(y_{t r, 2} \geq\left(y_{d l, 1}-0.35 \cdot y_{d l, 1}\right)\right) \\
0, \text { otherwise }
\end{array}\right.
$$

We also consider the following kinetic parameters, which are calculated between the ROIs of the first and the last images in a sequence of images stored for a given $t_{f}$ :

- Horizontal fall velocity, $v_{h}$, describes a ratio between the real horizontal velocity (equal to the factual height change between the first and the last images' ROIs) and the maximum possible horizontal velocity (equal to the height of the ROI of the first image).

$$
v_{h}=\frac{\sqrt{\left[\left(x_{t r, 1}-x_{d l, 1}\right)-\left(x_{t r, n}-x_{d l, n}\right)\right]^{2}}}{\sqrt{w^{2}+h^{2}}}
$$

- Vertical fall velocity, $v_{v}$, describes a ratio between the real vertical velocity (equal to the factual width change between the first and the last images' ROIs) and the maximum possible vertical velocity (equal to the width of the ROI of the first image).

$$
v_{v}=\frac{\sqrt{\left[\left(y_{t r, n}-y_{d l, n}\right)-\left(y_{t r, n}-y_{d l, n}\right)\right]^{2}}}{\sqrt{w^{2}+h^{2}}}
$$

Notice that the denominator in formulae (6) and (5) is the maximum possible fall velocity.

\subsection{Fuzzy System Design}

After the previous parameters (fall indicators) have been calculated, fuzzy logic is used for fall detection to determine the ranges of the fall indicators and to classify the possible fall patterns. Fuzzy logic eases the problem of fall detection, on the one hand, and facilitates recognition of fall patterns, on the other. Fig. 1 provides a schematic view of the proposed fuzzy inference system which executes as follows. Initially, input crisp variables are transformed into fuzzy sets within the "Fuzzification block". Next, the 


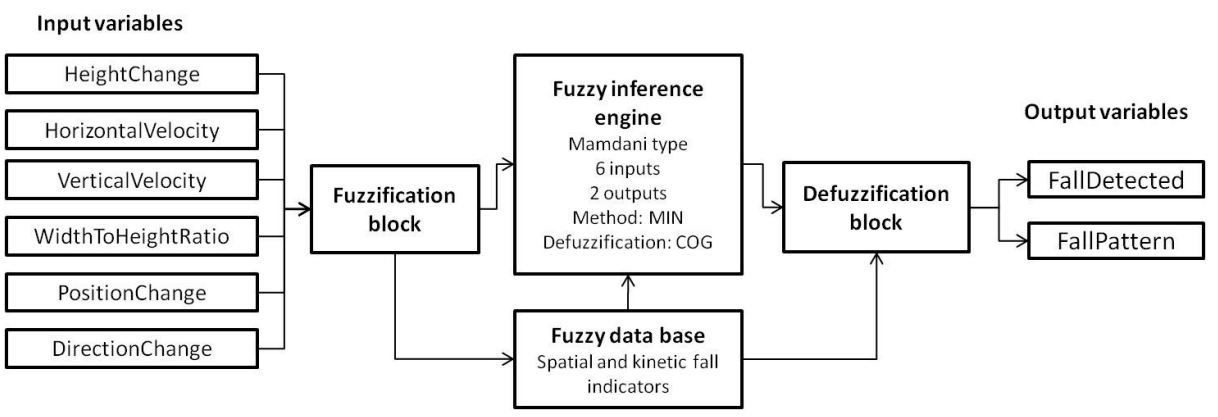

Fig. 1. Fuzzy inference system for fall pattern recognition

"Fuzzy inference engine" simulates the reasoning process by making fuzzy inference on the inputs and fuzzy "IF-THEN" rules that are stored within the "Knowledge base". The "Knowledge base" includes fuzzy rules and cases from "Fuzzy data base". Lastly, the fuzzy set obtained by the "Fuzzy inference engine" is transformed into crisp values corresponding to the output variables.

The fuzzy logic library "fuzzylite" (see http: / / code.google.com/p/fuzzy $-1 i t e /$ ), which provides a set of classes and methods for fuzzy inference system creation and manipulation, is used. The fuzzy system is codified in agreement with the Fuzzy Control Language which is a standard for Fuzzy Control Programming published by the International Electrotechnical Commission (IEC) (see http: / / www . ansi. org/). The linguistic variables describe the spatial and kinetic properties of ROIs corresponding to humans. The proposed fuzzy model for fall detection includes six input linguistic variables corresponding to the indicators described, that is, HeightChange $(\Delta h)$, HorizontalVelocity $\left(v_{h}\right)$, Vertical Velocity $\left(v_{v}\right)$, WidthToHeightRatio $\left(R_{w h}\right)$, PositionChange $(\Delta p)$ and DirectionChange $\left(d i r_{f}\right)$, and two output linguistic variables called FallDetected and FallPattern.

The fuzzy sets for input linguistic variables HeightChange, HorizontalVelocity, VerticalVelocityand WidthToHeightRatio include three fuzzy terms, namely LOW, MEDIUM and HIGH. Precisely, Fig. 2 shows the linguistic variables. Variable HeightChange is calculated as the relation of the initial height to the final height of the ROI. As stated in [9], the width of a human's body is around 25 percent of his/her height. Taking into account different human builds, we have experimentally decided to set up a value of 30 percent of a ROI's height as the highest boundary for the HIGH term of linguistic variable HeightChange. Variables VerticalVelocity and HorizontalVelocity are calculated as the relation of the real velocity to the maximum possible velocity through formulae (6) and (5). Thus, in case of a very quick fall with velocity equal or close to the maximum, the following expressions $v_{v} \approx 1.25$ and $v_{h} \approx 1.25$ are gotten. On the contrary, the change of velocity is minimal when velocity is $\approx 0.0$. The terms for these linguistic variables have the following ranges: LOW $\in[0 \ldots 0.35]$, MEDIUM $\in[0.25 \ldots 0.65]$ and $\mathrm{HIGH} \in[0.6 \ldots 1.25]$.

The Width ToHeightRatio variable ranges from 0.0 to 6.0. Since a ROI corresponding to a person in a strengthened position is longer in height than in width, the term 

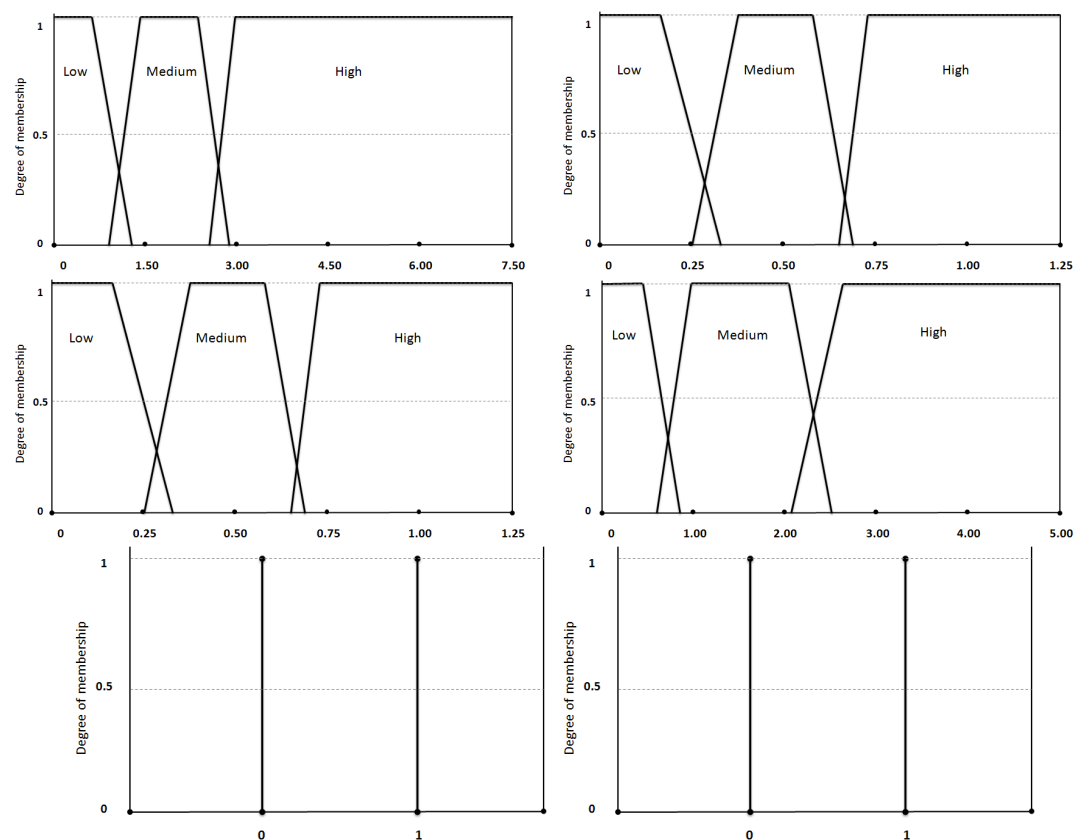

Fig. 2. Representation of linguistic variables HeightChange (top left), VerticalVelocity (top right), HorizontalVelocity (center left), WidthToHeightRatio (center right), PositionChange (bottom left) and DirectionChange (bottom right)

LOW $\in[0 \ldots 0.8]$, the term MEDIUM $\in[0.7 \ldots 2.5]$ stands for a situation where a ROI belongs to a fallen person, or, alternatively, to a sitting or bent forward person. Finally, the term HIGH $\in[2.1 \ldots 6.0]$ describes a ROI of a lying person. The PositionChange and DirectionChange variables are represented with crisp sets " 0 " and " 1 " for each variable. Output linguistic variable FallDetected is represented with fuzzy terms "NO" $\in[0 \ldots 45.0]$ and "YES" $\in[35.0 \ldots$. 100] (see Fig. 3). The FallPattern linguistic variable is also shown in Fig. 2 and includes a set of seven crisp values which correspond to the following fall patterns:

(1) Falling backward or forward from a standing position.

(2) Falling to the right from a standing position.

(3) Falling to the left from a standing position.

(4) Falling backward of forward from a sitting position.

(5) Falling on to the right from a sitting position.

(6) Falling on to the left from a sitting position.

(7) Falling backward of forward from a lying position.

The fuzzy system reasons about the state of a person. Thus, the first output variable, FallDetected, contains the resulting response of the system with two membership values corresponding to the confidence of a fall. The second output variable, FallPattern, generates a crisp value ranged from 1 to 7 , which indicates the detected fall pattern. 

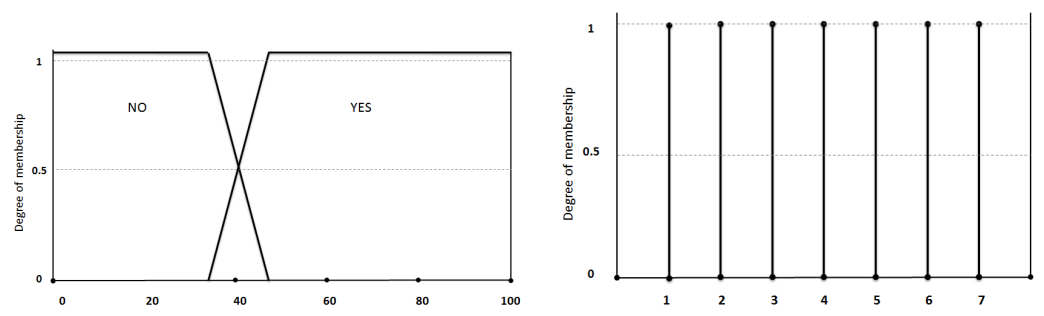

Fig. 3. Representation of output linguistic variables FallDetected (left) and FallPattern (right)

\section{Data and Results}

The following experiments were performed to validate the proposal. The experiments were carried out on an Intel Core i7 PC with 3 GB RAM under Windows XP. The video sequences were recorded with a FLIR A320 infrared camera at a resolution of $720 \times 480$ pixels. Nine series containing a single person were recorded to test the approach. The results of possible fall types are shown in Table 1 . The experiments cover the objectives established: static and dynamic falls from standing and sitting positions, falls from a lying position and a false fall. The capture of the videos was performed with an interval of 200 milliseconds, the fall time was set to 1.2 seconds. Thus, each six consecutive frames were tested for detecting a fall. Table 1 also contains the values for evaluating the falls.

The columns of Table 1 contain the values for the input and output variables of the fuzzy system, that is, $w h R, \Delta h, v_{h}, v_{v}, \operatorname{dir}_{f}$ and $\Delta p$ for each time period of a possible fall $t_{f}$. The column FallDetected shows the response of the model corresponding to the confidence of a fall, and the FallPattern column contains the number associated to the type of recognized fall pattern. Fig. 4 shows the images offering the falls detected in the experiment. There are red and blue bounding boxes in the images. The red bounding box represents the ROI at the end of the fall time, $t_{f}$, and the blue bounding box corresponds to the initial ROI. So, fall indicators are calculated using the red and the blue bounding boxes.

Detection of Static and Dynamic Falls from a Standing Position. Static falls include falling from a standing still position and dynamic falls occurs when a person is moving (walking, running, and so on) before the fall. Falls from a standing position are characterized by high horizontal velocity and height change. Also the width-to-height ratio changes significantly. We used a dynamic fall from a walking position to test the proposal. This type of fall has the same characteristics as a static fall from the standing position, though velocity values are higher than in case of static falls. Fig. 4a, Fig. $4 \mathrm{~b}$, and Fig. 4k show fall instants for three statical falls: for a person falling backward, falling forward and falling to the left. The fall is detected with a highest value 67.5, which corresponds to the "YES" fuzzy term of the FallDetected linguistic variable. FallPattern is recognized correctly as "1", which corresponds to "Falling backward or forward from a standing position". The second fall is again recognized as "1", although the fuzzy response for the variable FallDetected descends from 65.44 to 35.81 , and, 
Table 1. Experimental results for fall detection and recognition of fall patterns

\begin{tabular}{|c|c|c|c|c|c|c|c|c|}
\hline & whR & $\Delta h$ & $v_{h}$ & $v_{v}$ & $d i r_{f}$ & $\Delta p$ & FallDetected & FallPattern \\
\hline $\begin{array}{l}\text { STATIC FALL } \\
\end{array}$ & 1.14 & 1.30952 & 0.055 & 0.583 & 0 & 0 & 67.5 & \multirow{3}{*}{1} \\
\hline "STANDING" POSITION & 1.44 & 1.25 & 0.071 & 0.571 & 0 & 1 & 67.5 & \\
\hline Falling backward & 1.75 & 1.40127 & 0.087 & 0.610 & 0 & 0 & 67.5 & \\
\hline STATIC FALL & 1.19 & 1.019 & 0.215 & 0.914 & 0 & 0 & 65.44 & \multirow{5}{*}{1} \\
\hline "STANDING" POSITION & 1.32 & 1.212 & 0 & 0.177 & 1 & 0 & 38.65 & \\
\hline \multirow[t]{3}{*}{ Falling forward } & 1.43 & 1.125 & 0 & 0.322 & 0 & 1 & 39.50 & \\
\hline & 1.53 & 1.117 & 0.037 & 0.560 & 0 & 1 & 35.81 & \\
\hline & 1.68 & 1.075 & 0.560 & 0.560 & 0 & 0 & 67.5 & \\
\hline STATIC FALL & 1.5 & 1.732 & 0.024 & 0.332 & 0 & 0 & 67.49 & \multirow{8}{*}{3} \\
\hline “STANDING” POSITION & 2.54 & 1.165 & 0.544 & 0.072 & 0 & 0 & 66.43 & \\
\hline \multirow[t]{6}{*}{ Falling to the left } & 2.95 & 1.180 & 0.550 & 0.084 & 0 & 0 & 67.5 & \\
\hline & 3.27 & 1.076 & 0.847 & 0.096 & 0 & 0 & 53.59 & \\
\hline & 2.93 & 1.345 & 0.544 & 0.151 & 0 & 0 & 67.5 & \\
\hline & 3.31 & 1.464 & 0.544 & 0.163 & 0 & 0 & 67.5 & \\
\hline & 3.38 & 1.619 & 0.544 & 0.181 & 0 & 0 & 67.5 & \\
\hline & 4.53 & 1 & 0 & 0 & 1 & 0 & 67.5 & \\
\hline DYNAMIC FALL & 1.12 & 1.626 & 0.130 & 0.130 & 0 & 0 & 51.26 & \multirow{4}{*}{1} \\
\hline "STANDING" POSITION & 1.21 & 1.946 & 0.134 & 0.134 & 0 & 0 & 67.5 & \\
\hline \multirow[t]{2}{*}{ Falling forward } & 1.97 & 1.230 & 0 & 0 & 0 & 1 & 67.5 & \\
\hline & 2.4 & 1.671 & 0 & 0.215 & 0 & 0 & 67.5 & \\
\hline DYNAMIC FALL & 1.33 & 1.416 & 0.281 & 0.187 & 0 & 0 & 43.5 & \multirow{6}{*}{3} \\
\hline "STANDING" POSITION & 2.06 & 1.973 & 0.391 & 0.391 & 0 & 0 & 67.5 & \\
\hline \multirow{4}{*}{ Falling to the left } & 2.73 & 1.826 & 0.407 & 0.407 & 0 & 0 & 67.5 & \\
\hline & 2.71 & 1.826 & 0.317 & 0.317 & 0 & 0 & 67.5 & \\
\hline & 2.72 & 1.811 & 0.328 & 0.328 & 0 & 0 & 67.5 & \\
\hline & 2.72 & 1.811 & 0.340 & 0.340 & 0 & 0 & 67.5 & \\
\hline FALL & 2.99 & 1.040 & 0 & 0 & 0 & 0 & $\overline{58.05}$ & \multirow{6}{*}{4} \\
\hline “SITTING” POSITION & 3.0 & 1.051 & 0 & 0 & 0 & 0 & 58.52 & \\
\hline \multirow[t]{4}{*}{ Falling backward } & 2.84 & 1 & 0 & 0.012 & 0 & 0 & 56.44 & \\
\hline & 2.63 & 0.953 & 0.015 & 0.015 & 0 & 0 & 54.51 & \\
\hline & 3.89 & 0.986 & 0.016 & 0.016 & 0 & 0 & 55.97 & \\
\hline & 4.89 & 1.153 & 0.022 & 0.029 & 0 & 0 & 64.22 & \\
\hline FALL & 1.04 & 1.012 & 0.086 & 0.021 & 1 & 0 & 42.10 & \multirow{7}{*}{5} \\
\hline "SITTING” POSITION & 1.36 & 1.081 & 0.077 & 0.077 & 1 & 0 & 60.02 & \\
\hline \multirow[t]{5}{*}{ Falling to the right } & 1.43 & 0.905 & 0 & 0.045 & 1 & 0 & 53.59 & \\
\hline & 1.71 & 1.136 & 0.025 & 0.051 & 1 & 0 & 63.11 & \\
\hline & 2.1 & 1.794 & 0.226 & 0.226 & 1 & 0 & 67.5 & \\
\hline & 2.26 & 1.83 & 0.177 & 0.249 & 1 & 0 & 67.5 & \\
\hline & 2.31 & 1.776 & 0.135 & 0.226 & 1 & 0 & 67.5 & \\
\hline \multirow{6}{*}{$\begin{array}{c}\text { FALL } \\
\text { "LYING" POSITION }\end{array}$} & 3.03 & 1.212 & 0 & 0.182 & $\overline{11}$ & $\overline{11}$ & 38.65 & \multirow{6}{*}{7} \\
\hline & 3.02 & 1.125 & 0 & 0.317 & 0 & 1 & 39.50 & \\
\hline & 3.56 & 1.119 & 0.816 & 0.633 & 0 & 1 & 67.5 & \\
\hline & 4.14 & 1.271 & 1.014 & 0.713 & 0 & 1 & 67.5 & \\
\hline & 4.15 & 0.979 & 0 & 0.013 & 0 & 1 & 53.00 & \\
\hline & 4.22 & 1.052 & 0 & 0.032 & 0 & 1 & 58.03 & \\
\hline \multirow{7}{*}{$\begin{array}{l}\text { FALSE FALL } \\
\text { Kneeling }\end{array}$} & 2.1 & 2.165 & 1.141 & 0.439 & 0 & 0 & 32.5 & \\
\hline & 1.93 & 2.018 & 1.117 & 0.465 & 0 & 0 & 35.9 & \\
\hline & 1.95 & 1 & 0.602 & 0.200 & 0 & 0 & 33.7 & \\
\hline & 1.97 & 1.006 & 0.731 & 0.285 & 0 & 0 & 22.45 & \\
\hline & 2.04 & 1.040 & 0.731 & 0.303 & 0 & 0 & 0 & \\
\hline & 2.27 & 0.776 & 0.423 & 0.141 & 1 & 0 & 0 & \\
\hline & 1.92 & 0.678 & 0.507 & 0.282 & 1 & 0 & 0 & \\
\hline
\end{tabular}

finally, stabilizes in "YES" fuzzy term (67.5). The fall to the left is also detected and recognized correctly (marked as "3" in FallPattern variable). Fig. 4d shows frames with an instant of a fall for a walking person who is falling forward. The fall is detected correctly with the MEDIUM values for horizontal fall velocity, vertical fall velocity and 
(a)

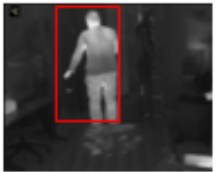

(b)

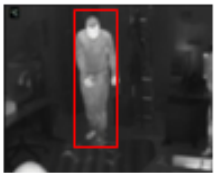

(c)

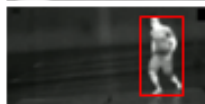

(d)

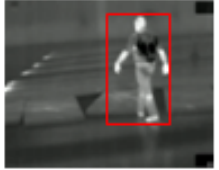

(e)

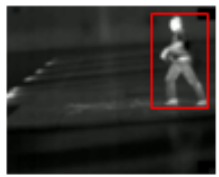

(f)

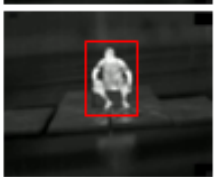

(g)

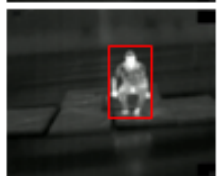

(h)

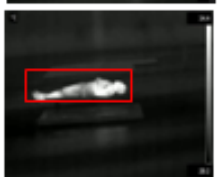

(i)

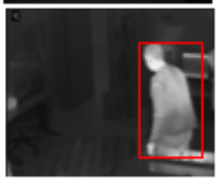

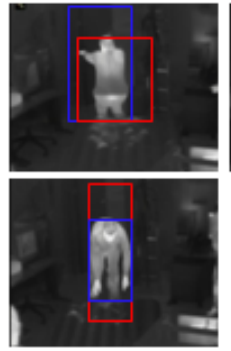
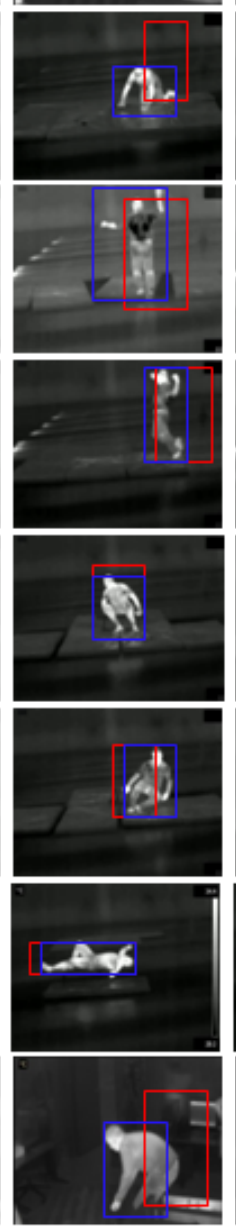
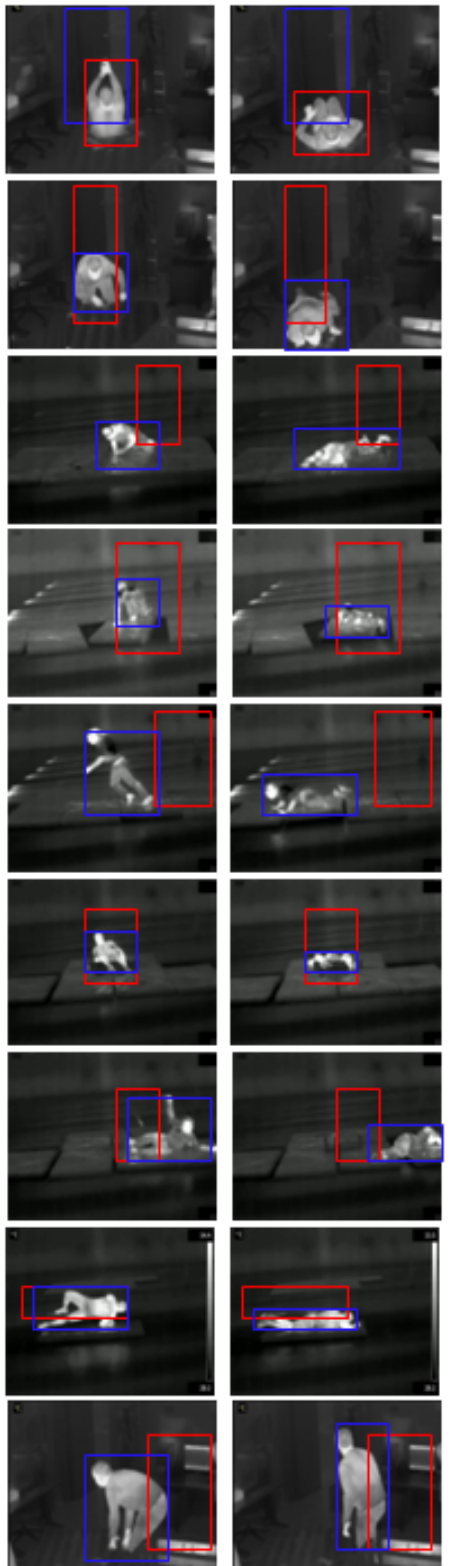

Fig. 4. True and false falls. Static falls from a standing position: (a) falling backward, (b) falling forward, (c) falling to the left. Dynamic falls from a standing position: (d) falling forward, (e) falling to the left. Falls from a sitting position: (f) falling backward, (g) falling to the right. (h) Falling from the lying position. (i) False fall: kneeling. 
width-to-height ratio parameters, as shown in Table 1 The dynamic fall to the left is correctly classified as " 3 ", that is "Falling to the left from a standing position" (see Fig. 4 e).

Detection of Falls from a Sitting Position. A couple of falls from a sitting position are provided in Fig. 4f and Fig. 4g. The first fall is characterized by a rapid increase of horizontal fall velocity and vertical fall velocity. The fall to the right is classified correctly, taking a value of " 1 " for the fall direction variable (see Table 1).

Detection of Falls from a Lying Position. This fall (see Fig. 4h) is recognized as "7" ("Falling to the left from a lying position"), as position change variable takes value "1" and WidthToHeightRatio input variable takes a value belonging to HIGH range (see Table 1).

Detection of False Falls. False falls are represented through a kneeling example. As a general rule, in case of a false fall linguistic variable width-to-height ratio uses to have LOW or MEDIUM values, and linguistic variables HorizontalVelocity and VerticalVelocity are rather LOW. The video sequence shown in this example does not include a detected fall (the variable FallDetected changes from 32.5 to 22.45 , which corresponds to the "NO" term). Fig. 4 shows an instant of the false fall. In compliance with the results given in Table 1, a fall is not detected in this case. This example demonstrates that the proposal is capable of differentiating true from false falls.

\section{Conclusions}

Elderly fall is an important problem which needs rapid fast and effective solutions. In this paper we have introduced a fuzzy-based fall detection model for humans segmented in infrared video. The geometrical characteristics of the region of interest corresponding to the detected person, and the velocity of the change of his/her bounding box serve as fall indicators. Additionally, the fuzzy model avoids certain limitations in parameter evaluation and makes smoother and more flexible decisions.

The proposal is incorporated into a fall detection system for the elderly, and then tested for a wide number of static and dynamic falls, including a test for false falls. The infrared camera system has shown to perform both efficiently at daytime and nighttime. Experimental results, which were carried out for static and dynamic falls, have demonstrated that the fall detection proposal is able to detect all these cases and to distinguish real falls from false ones.

Acknowledgements. This work was partially supported by FEDER and Spanish Ministerio de Economía y Competitividad under project TIN2010-20845-C03-01, Ministerio de Industria, Turismo y Comercio (Plan Avanza 2) under project TSI-0201002010-261, and Junta de Comunidades de Castilla-La Mancha under project PII2I090069-0994. 


\section{References}

1. Benocci, M., Tacconi, C., Farella, E., Benini, L., Chiari, L., Vanzago, L.: Accelerometerbased fall detection using optimized ZigBee data streaming. Microelectronics Journal 41(11), 703-710 (2010)

2. Doukas, C., Maglogiannis, I.: Emergency fall incidents detection in assisted living environments utilizing motion, sound, and visual perceptual components. IEEE Transactions on Information Technology in Biomedicine 15(2), 277-289 (2011)

3. Fernández-Caballero, A., Castillo, J.C., Rodríguez-Sánchez, J.M.: Human activity monitoring by local and global finite state machines. Expert Systems with Applications 39(8), 69826993 (2012)

4. Fernández-Caballero, A., Castillo, J.C., Serrano-Cuerda, J., Maldonado-Bascón, S.: Realtime human segmentation in infrared videos. Expert Systems with Applications 38(3), 25772584 (2011)

5. Fernández-Caballero, A., Castillo, J.C., Martínez-Cantos, J., Martínez-Tomás, R.: Optical flow or image subtraction in human detection from infrared camera on mobile robot. Robotics and Autonomous Systems 58(12), 1273-1283 (2010)

6. Khawandi, S., Daya, B., Chauvet, P.: Implementation of a monitoring system for fall detection in elderly healthcare. Procedia Computer Science 3, 216-220 (2011)

7. Klack, L., Möllering, C., Ziefle, M., Schmitz-Rode, T.: Future care floor: A sensitive floor for movement monitoring and fall detection in home environments. In: Proceedings of MobiHealth 2010, pp. 211-218 (2010)

8. Litvak, D., Zigel, Y., Gannot, I.: Fall detection of elderly through floor vibrations and sound. In: Proceedings of the 30th Annual International Conference of the IEEE Engineering in Medicine and Biology Society, pp. 4632-4635 (2008)

9. Loomis, A.: Figure Drawing for All it's Worth. Viking Adult (1943)

10. Mo, H.C., Leou, J.J., Lin, C.S.: Human behavior analysis using multiple $2 \mathrm{~d}$ features and multi category support vector machine. In: Proceedings of the IAPR Conference on Machine Vision Applications, pp. 46-49 (2009)

11. Moreno-Garcia, J., Rodriguez-Benitez, L., Fernández-Caballero, A., López, M.T.: Video sequence motion tracking by fuzzification techniques. Applied Soft Computing 10(1), 318-331 (2010)

12. Pellegrini, S., Iocchi, L.: Human posture tracking and classification through stereo vision and 3d model matching. EURASIP Journal on Image and Video Processing, 1-12 (2008)

13. Rimminen, H., Lindström, J., Linnavuo, M., Sepponen, R.: Detection of falls among the elderly by a floor sensor using the electric near field. IEEE Transactions on Information Technology in Biomedicine 14(6), 1475-1476 (2010)

14. Rojas-Albarracín, G., Carbajal, C.A., Fernández-Caballero, A., López, M.T.: Skeleton simplfication by key points identfication. In: Proceedings of the 2nd Mexican Conference on Pattern Recognition, pp. 30-39 (2010)

15. Thome, N., Miguet, S., Ambellouis, S.: A real-time, multiview fall detection system: A LHMM-based approach. IEEE Transactions on In Circuits and Systems for Video Technology 18(11), 1522-1532 (2008)

16. Wei, X., Chai, J.: Modeling $3 \mathrm{~d}$ human poses from uncalibrated monocular images. In: Proceedings of the IEEE International Conference on Computer Vision, pp. 1873-1880 (2009)

17. Yu, X.: Approaches and principles of fall detection for elderly and patient. In: Proceedings of the 10th IEEE HealthCom 2008, pp. 42-47 (2008)

18. Zweng, A., Zambanini, S., Kampel, M.: Introducing a Statistical Behavior Model into Camera-Based Fall Detection. In: Bebis, G., Boyle, R., Parvin, B., Koracin, D., Chung, R., Hammoud, R., Hussain, M., Kar-Han, T., Crawfis, R., Thalmann, D., Kao, D., Avila, L. (eds.) ISVC 2010. LNCS, vol. 6453, pp. 163-172. Springer, Heidelberg (2010) 